\title{
Performances Économiques Comparées Des Unités De Pêche En Lagune Et Des Alternatives Agricoles Et Aquacoles À Grand-Lahou (Côte d'Ivoire)
}

\author{
Balle Ségbé Guy Romaric (Doctorant) \\ Université Peleforo GON COULIBALY Côte d'Ivoire \\ Ouattara Allassane (Professeur) \\ Université Nangui Abrogoua \\ Vanga Adja Ferdinand (Professeur) \\ Université Peleforo GON COULIBALY Côte d'Ivoire \\ Gourene Germain (Professeur) \\ Université Nangui Abrogoua Côte d'Ivoire
}

doi: 10.19044/esj.2017.v13n13p211 URL:http://dx.doi.org/10.19044/esj.2017.v13n13p211

\begin{abstract}
The lagoons are high biological and economic potential areas exploited by thousands of fishermen. Otherwise, the persistent use of pesticides in fisheries may be perceived as a response of fishermen to lower yields and, consequently, to reduced income from fishing activities. In this context, the hypothesis of a lack of profitability concerning the activity of professional fishermen was therefore raised. To reach this goal, a market survey, investments and fish landings were carried out in order to analyze the profitability and economic performance of this activity. It recorded 5227 catch exits from a sample of 35 fixed fishermen in 7 villages. These were followed 15 times a month for 12 months whenever possible. In practice, approximately $60 \%$ of the suspected activity of professional fishermen has been observed. After elaborating the annual operating accounts and calculating the performance ratios, it appears that the lagoon fishing activity of Grand-Lahou is profitable and competitive compared to the alternative economic activities of the study area. However, performance is not homogeneous between villages. This suggests a fishing-related effect on fishers' performance. In addition, it is the Liza falcipinnis and Chrysichthys nigrodigitatus species that significantly influence the value of production and hence income.
\end{abstract}

Keywords : Artisanal fisheries, income, competitiveness, Lagoon, Ivory Coast 


\section{Résumé}

Les lagunes sont des milieux à fort potentiel biologique et économique exploités par des milliers de pêcheurs. Par ailleurs, l'utilisation persistante de pesticides dans la pêche peut être perçues comme une réponse des pêcheurs à la baisse des rendements et, par conséquent, à la réduction des revenus tirés de l'activité de pêche. Dans ce contexte, l'hypothèse d'un défaut de rentabilité de l'activité des pêcheurs professionnels a donc été posée. Pour ce faire, une enquête sur le marché, les investissements et les débarquements y a été menée en vue de procéder à l'analyse de la rentabilité et des performances économiques de cette activité. Elle a permis d'enregistrer 5227 sorties de capture à partir d'un échantillon de 35 pêcheurs fixes repartis dans 7 villages. Ceux-ci ont été suivis 15 fois par mois pendant 12 mois lorsque cela était possible. Dans la pratique, ce dispositif a permis d'observer environ 60 \% de l'activité présumée des pêcheurs professionnels. Après avoir élaboré les comptes d'exploitation annuels et calculé les ratios de performances, il ressort que l'activité de pêche en lagune de Grand-Lahou est rentable et compétitive par rapport aux activités économiques alternatives de la zone d'étude. Toutefois, les performances ne sont pas homogènes selon les villages. Cela laisse entrevoir un effet lié à l'aire de pêche sur le rendement des pêcheurs. Par ailleurs, ce sont les espèces Liza falcipinnis et Chrysichthys nigrodigitatus qui influencent significativement la valeur de la production et partant, des revenus.

Mots clés : Pêche artisanale, revenus, compétitivité, lagune, Côte d’Ivoire

\section{Remerciements}

La réalisation de ce papier n'aurait pu être possible grâce à la coopération effective des communautés de pêcheurs des localités enquêtées. Nos remerciements vont également à l'endroit de Philippe Cecchi de l’Université de Montpellier ; de Christian Chaboud, Nicolas Bez, Monique Simier et Gilles Domalain à l'UMR MaRBEC de l'IRD de Sète (France) pour leur contribution à l'analyse des données et pour leurs conseils avisés.

\section{Introduction}

Parmi les ressources naturelles renouvelables, le poisson joue un rôle fondamental pour les hommes notamment en tant que nourriture, qu'ils soient pêchés dans la nature ou élevés en pisciculture. Selon FAO (2014), les pêches de capture et l'aquaculture ont produit en 2012 environ 110 millions de tonnes de poisson destiné à l'alimentation, situant l'offre apparente par habitant à 16,7 kilogrammes, soit l'une des plus fortes jamais enregistrées. Dans cet ensemble, 10 millions de tonnes, soient 10 milliards de dollars US 
proviennent des captures dans les eaux intérieures. Parmi ces milieux de vie, la lagune représente un cadre naturel particulier, caractérisé par une grande diversité d'espèces marines, saumâtres ou continentales. Elle constitue de ce fait un milieu à fort potentiel biologique et économique (Albaret, 1994 ; Charles-Dominique, 1993 ; Anoh, 2010).

La Côte d'Ivoire compte quatre (4) lagunes dont celle de GrandLahou où se pratique une pêche individuelle avec les principaux engins qui sont : le filet maillant, l’épervier, la palangre et la nasse. Cette particularité réduit la capacité de contrôle des autorités coutumières et administratives sur les engins et les méthodes de pêche. Des enquêtes préliminaires à la présente étude, réalisées en 2008 auprès des pêcheurs, révèlent une baisse des captures journalières qui réduirait les revenus des pêcheurs. En général, l'argument de la surexploitation des pêcheries tropicales est le plus souvent avancé pour expliquer la baisse de la productivité des plans d'eau (Pauly and al., 2002). En pratique, selon Allison and Ellis (2001) et Cinner and al. (2008), les pêcheurs développent une gamme de stratégies et de réponses adaptatives pour faire face aux fluctuations des rendements. Entre autres, ils peuvent alors passer temporairement ou de manière alternée à une autre activité génératrice de revenu, tenter de masquer le déclin des rendements avec une augmentation de l'effort ou utiliser des moyens de captures plus efficaces ou plus destructeurs (Pauly, 1990 ; Mc Clanahan and al., 2005). La dernière alternative est récurrente en lagune de Grand-Lahou, réputée depuis quelques années pour ses méthodes prohibées dont l'usage de pesticides et d'engins aux dimensions non conventionnelles (Sankaré et al., 1994 ; Bleu, 2015 ; Kouakou et al., 2015 ; Anoh, 2007). Selon Polunin and al. (1996), les difficultés rencontrées dans la gestion des pêcheries tropicales résideraient dans la situation de pauvreté des pêcheurs les conduisant à des pratiques illégales en vue d'améliorer les résultats économiques de leur unité de pêche. Cet état de pauvreté rendrait inefficace les politiques de régulation de l'activité de pêche et créerait une attraction particulière pour les activités économiques alternatives; notamment l'agriculture et l'aquaculture. La présente étude consiste à l'analyse économique de la pêche artisanale en lagune de Grand-Lahou afin d'évaluer les performances économiques des unités de pêche au filet maillant en opposition avec les alternatives agricoles et aquacoles de la zone d'étude. Cette recherche s'articule autour d'une approche méthodologique pluridisciplinaire, des résultats de terrain et de leur discussion.

\section{Approche methodologique}

\section{Choix des villages échantillonnés}

Au total, 7 villages sur les 26 contigus à la lagune ont été identifiés pour abriter les enquêtes. Ainsi, les localités de Lahou-Kpanda, Braffedon, 
N'Guessandon et de Noumouzou encadrant le grau de Grand-Lahou sur la lagune Tagba, la localité de Mackey sur la lagune Mackey, la localité de Tioko sur la lagune Tadio et la localité de Kokou sur la lagune Niouzoumou ont été choisies.

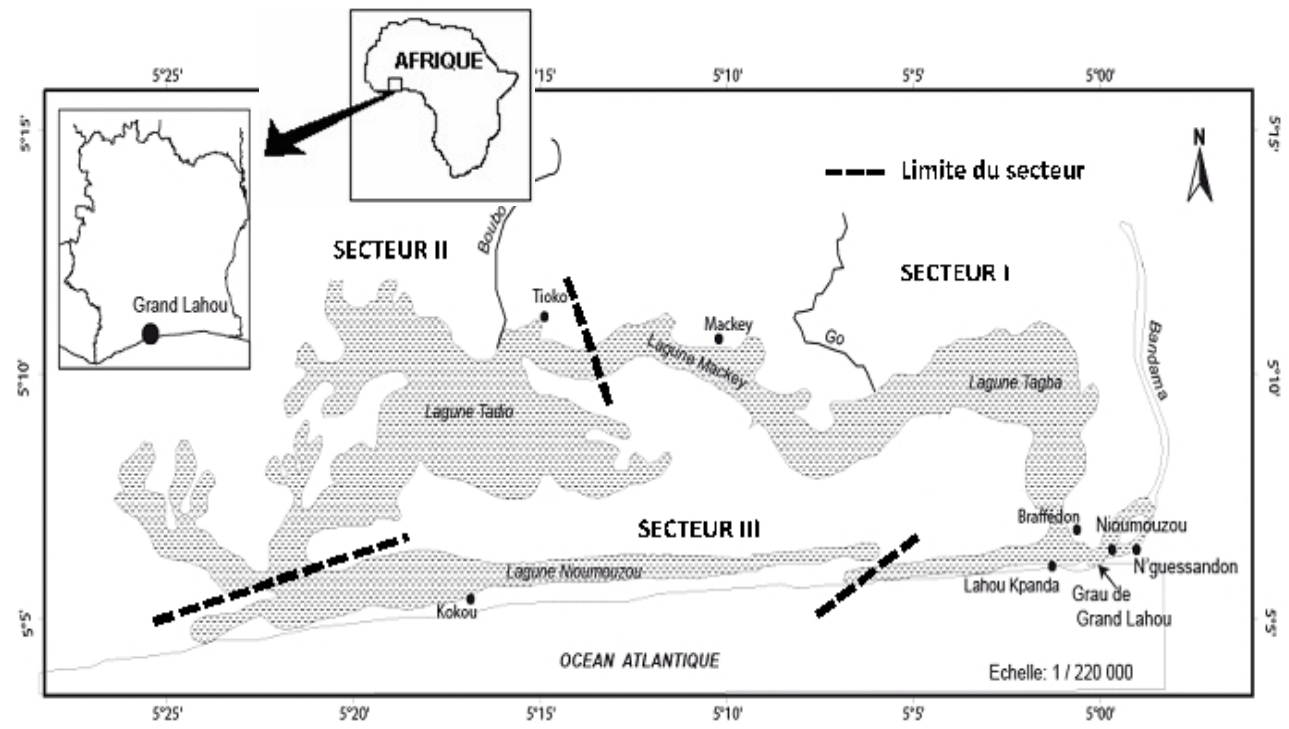

Figure 1 : Localisation des sept (7) village-stations échantillonnés

\section{Méthode d'échantillonnage}

Pour accroître la fiabilité des données quantitatives soumises à de nombreuses sources de variation, l'échantillonnage par panel est bien indiqué (Aktouf, 1987). Ainsi, cinq (5) pêcheurs professionnels (nombre d'années d'expérience supérieure à 10 ans) ayant le filet maillant comme engin principal, ont été choisis parmi les plus réguliers dans l'activité de pêche. Leur activité a été recensée pendant 15 jours par mois, lorsque cela était possible. L’âge, l'expérience (nombre d'années passé dans l'activité), le niveau d'étude et le nombre de personnes à charge du pêcheur ont aussi été notés. Cette enquête a couvert toutes les semaines, du 5 janvier 2010 au 29 décembre 2010. Au total, douze (12) mois d'échantillonnage ont été respectés en vue de couvrir un cycle complet de pêche (Charles-Dominique et al., 1980; Gérard et Gréber, 1985; Laë, 1992). Dans le dispositif, le pas de temps est le jour et l'effort de pêche est la sortie de pêche d'un professionnel au métier du filet maillant. Le système déployé a permis d'enregistrer 5227 sorties de pêche correspondant à $83 \%$ des 6300 initialement visées par le protocole d'enquête et $60 \%$ du taux d'activité moyen en pêche continentale tel qu'indiquée par UEMOA (2013).

Dans la pratique, un suivi des débarquements a été réalisé et les prix de vente au débarcadère des principales familles ont été relevés dans chaque localité en FCFA/Kg ou en FCFA/Tas. La détermination des captures a été 
faite jusqu’à l’espèce lorsque cela était possible selon la clé proposée par Levèque et al. (1992) et Paugy et al. (2004).

\section{Méthode d'analyse économique}

Cette analyse combine l'approche socio-économique avec l'approche comptable conformément aux travaux de Kébé (1997) et Turay et Verstralen (1997). L’approche socio-économique vient en complément de la méthode comptable en vue d'intégrer les valeurs des facteurs qui ne font pas l'objet d'un décaissement dans une évaluation globale. Cela revient à considérer ces facteurs de production à leur coût d’opportunité (Levrel et al., 2012). Les coûts fixes sont constitués ici de : l'amortissement des engins de pêche et de la pirogue et des intérêts sur avances (placement à 3\% de taux d’intérêt). Les coûts variables sont composés des valeurs locatives de la main d'œuvre et de la pirogue et des frais de réparation. Les deux premiers postes sont estimés à leur coût d'opportunité car il y a une non recette due à la renonciation au loyer de la main d'œuvre et de la pirogue vu que le marché existe.

Les formules des paramètres d'analyse sont indiquées ci-après (Bridier et Michaïlof, 1995) :

Revenu brut ou chiffre d'affaire ou valeur de la production (1) :

$$
R b=\sum_{i=1}^{n} P_{i} Q_{i}
$$

$\mathrm{Rb}=$ Revenu brut ou valeur de la production (VP) $\mathrm{n}=$ nombre d'espèces pêchées

$$
\mathrm{P}_{\mathrm{i}}=\text { Prix de vente du kg de l'espèce } \mathrm{i} \quad \mathrm{Q}_{\mathrm{i}}=\text { Quantité de }
$$

l'espèce i vendue

Revenu net (2)

$$
\begin{aligned}
& R n=\sum_{i=1}^{n} P_{i} Q_{i}-(C V+C F) \text { Ou } R n=\sum_{i=1}^{n} P_{i} Q_{i}-C T \quad \text { (2) } \\
& \text { Rn = Revenu net } \quad \mathrm{CV}=\text { Coûts variables } \mathrm{CF}=\text { Coûts fixes } \\
& \mathrm{CT}=\text { Coûts totaux }
\end{aligned}
$$

Valeur Ajoutée Nette (VAN) (3)

VAN = Valeur de la production - Frais de Réparation Amortissement

Taux de valeur ajoutée (4)

$$
\mathrm{TVA}=\frac{\text { VAN }}{\text { Valeur de la production }}
$$

Revenu du travail (5)

Revenu du travail $=$ résultat net + rémunération de la main d’œuvre 
Taux de rentabilité du capital investi (TRCI)

$T R C I=\frac{\text { Résultat net annuel }}{\text { Montant capital investi }}$

Délai de récupération du capital investi (DRCI) ou effort de pêche critique de rentabilité (7)

DRCI $=\frac{\text { Montant capital investi }}{\text { Résultat net annuel }} \times 252$ Sorties

Coût de production du kilo de poisson débarqué (CPKPD)

$C P K P D=\frac{\text { Coûts totaux }}{\text { Poids total débarqué }}$

\section{Analyse statistique}

La moyenne, l'écart type et le coefficient de variation ont été calculés pour les valeurs analysées. Ces statistiques ont permis de décrire et de faire ressortir les limites des variations des paramètres étudiés (Zar, 1999). L’ANOVA a été utilisée pour comparer les variances des valeurs entre les villages. Dans le but de satisfaire les hypothèses de normalité et d'homoscédasticité de l'ANOVA, les valeurs ont été converties par la fonction $\log (x+1)$ avant d'être analysées ; dans cette formule, $x$ représente la valeur à transformer. A la suite de l'analyse des variances, le test de comparaison multiple des moyennes de Student-Newman-Keuls (SNK) a été appliqué pour identifier les objets ayant des variances homogènes (Zar, 1999 ; Francini-Filho and Moura, 2008). Par ailleurs, le test de rang de Spearman a permis d'établir la matrice de corrélation entre les caractéristiques sociodémographiques du pêcheur, les moyens investis, les résultats financiers et les niveaux de performances des unités de pêches.

\section{Méthode de comparaison des résultats}

Les spéculations retenues pour la comparaison sont: le piment (Capsicum annum), l'oignon (Allium cepa), la tomate (Salanum lycopersicum), le riz (Oryza sativa) et la pépinière de l’hévéa (Hevea brasiliensis) pour les cultures végétales. Les productions aquacoles concernent: l'élevage en étang d'Oreochromis niloticus et de Clarias gariepinus. Les paramètres de performances économiques ont été calculés à partir des comptes d'exploitation prévisionnels. Pour certaines productions végétales, les prévisions sont établies aussi bien en mode extensif qu'en mode intensif. Les estimations sont faites sur une base annuelle afin de rendre possible la comparaison des soldes et des ratios (cycles/an). 


\section{Resultats}

Les résultats des différents traitements sont contenus dans les tableaux ci-après. Les valeurs monétaires sont exprimées en Francs de la Communauté Financière Africaine (FCFA).

Tableau 1 : Comptes d'exploitation des unités de pêches au filet maillant par village, par pêcheur professionnel et par an

\begin{tabular}{|c|c|c|c|c|c|c|c|c|}
\hline Villages & $\begin{array}{l}\text { Moy VP } \\
\text { SD VP } \\
\end{array}$ & $\begin{array}{l}C V_{V P} \\
(\%)\end{array}$ & $\begin{array}{c}\text { Moy CVa } \\
\text { SD CVa }\end{array}$ & $\begin{array}{l}C_{C V} \\
(\%)\end{array}$ & $\begin{array}{l}\text { Moy CFi } \\
\text { SD CFi }\end{array}$ & $\begin{array}{l}C_{\text {CFi }} \\
(\%)\end{array}$ & $\begin{array}{l}\text { Moy RN } \\
\text { SD RN }\end{array}$ & $\begin{array}{l}C_{\mathrm{RN}} \\
(\%)\end{array}$ \\
\hline Braffedon & $\begin{array}{l}2 \\
460155^{\mathrm{a}} \\
\pm 782992\end{array}$ & 32 & $\begin{array}{l}940237^{a} \\
\pm 23546\end{array}$ & 3 & $\begin{array}{l}333057^{\mathrm{ac}} \\
\pm 99493\end{array}$ & 30 & $\begin{array}{l}1186968^{\text {ad }} \\
\pm 715151\end{array}$ & 60 \\
\hline Kokou & $\begin{array}{l}2 \\
643547^{\mathrm{a}} \\
\pm 283972\end{array}$ & 11 & $\begin{array}{l}957137^{a} \\
\pm 16396\end{array}$ & 2 & $\begin{array}{l}438696^{\mathrm{a}} \\
\pm 95097\end{array}$ & 22 & $\begin{array}{l}1247714^{\text {abd }} \\
\pm 290811\end{array}$ & 23 \\
\hline Lahou & $\begin{array}{l}4 \\
213997^{\mathrm{a}} \\
\pm 664807\end{array}$ & 16 & $\begin{array}{l}946714^{\mathrm{a}} \\
\pm 10499\end{array}$ & 1 & $\begin{array}{l}398088^{a} \\
\pm 72029\end{array}$ & 18 & $\begin{array}{l}2869178^{c} \\
\pm 647539\end{array}$ & 23 \\
\hline Mackey & $\begin{array}{l}1 \\
200126^{\mathrm{b}} \\
\pm 857584\end{array}$ & 71 & $\begin{array}{l}896666^{\mathrm{b}} \\
\pm 13971\end{array}$ & 2 & $\begin{array}{l}123192^{b} \\
\pm 58469\end{array}$ & 47 & $\begin{array}{l}180174^{\mathrm{d}} \\
\pm 793651\end{array}$ & 440 \\
\hline N'guessandon & $\begin{array}{ll}3 & 005 \\
176^{\mathrm{a}} & \\
\pm 238 & 468\end{array}$ & 8 & $\begin{array}{l}544329^{c} \\
\pm 17307\end{array}$ & 3 & $\begin{array}{l}470498^{\mathrm{a}} \\
\pm 128112\end{array}$ & 27 & $\begin{array}{l}1990591^{\text {ac }} \\
\pm 228328\end{array}$ & 11 \\
\hline Noumouzou & $\begin{array}{l}3 \\
257^{\text {a }} \\
\pm 332696\end{array}$ & 10 & $\begin{array}{l}551275^{c} \\
\pm 10496\end{array}$ & 2 & $\begin{array}{l}522056^{a} \\
\pm 50147\end{array}$ & 10 & $\begin{array}{l}2312374^{\mathrm{bc}} \\
\pm 292330\end{array}$ & 13 \\
\hline Tioko & $\begin{array}{ll}3 & 016 \\
496^{\mathrm{a}} & \\
\pm 239 & 537\end{array}$ & 8 & $\begin{array}{l}919346^{\mathrm{ab}} \\
\pm 13638\end{array}$ & 1 & $\begin{array}{l}211392^{\mathrm{c}} \\
\pm 71933\end{array}$ & 34 & $\begin{array}{l}1885734^{\text {ac }} \\
\pm 255940\end{array}$ & 14 \\
\hline Probabilités & $\mathrm{P}<0,05$ & & $\mathrm{P}<0,05$ & & $\mathrm{P}<0,05$ & & $\mathrm{P}<0,05$ & \\
\hline Moy.LGL & $\begin{array}{l}2846108 \\
\pm 999271\end{array}$ & 35 & $\begin{array}{r}822243 \\
\pm 188520\end{array}$ & 23 & $\begin{array}{r}356711 \\
\pm 144296\end{array}$ & 40 & $\begin{array}{l}1667533 \\
\pm 947893\end{array}$ & 57 \\
\hline
\end{tabular}

Moy : Moyenne SD : écart type CV : Coefficient de variation VP : Valeur de la production CVa: Charges variables $\mathrm{CFi}$ : Charges fixes RN : Résultat net ; LGL : Lagune de Grand-Lahou Les valeurs portant la même lettre en exposant ont des variances homogènes

Le tableau 1 indique les comptes d'exploitation comparés des unités de pêche au filet maillant par village, par pêcheur sur une année. Les valeurs monétaires de la production des différents villages sont statistiquement égales à l'exception de celle du village de Mackey. Cette localité présente le chiffre d'affaire moyen le plus faible des villages stations. La valeur de la production la plus importante est observée à Lahou. Les coefficients de variations des chiffres d'affaire varient de 8 à $32 \%$. Les productions monétaires annuelles ont des valeurs homogènes dans 5 localités $\left(\mathrm{CV}_{\mathrm{VP}}<\right.$ 25\%). A l'inverse, Mackey et Braffedon connaissent une forte variation des chiffres d'affaire annuels $\left(\mathrm{CV}_{\mathrm{VP}}>25 \%\right)$.

Le solde du compte d'exploitation annuel est positif dans la totalité des unités de pêche au filet maillant. L'activité de pêche est donc rentable pour ce métier. Le résultat net est le plus élevé à Lahou et le plus bas à Braffedon et à Mackey avec des coefficients de variation supérieurs à $50 \%$. 
Tableau 2 : Valeur ajoutée nette (VAN) annuelle par pêcheur professionnel par village.

\begin{tabular}{|c|c|c|c|c|c|}
\hline Villages & $\begin{array}{l}\text { Moy VP } \\
\text { SD VP }\end{array}$ & $\begin{array}{c}\text { Moy } \\
\text { Réparation }\end{array}$ & $\begin{array}{c}\text { Moy } \\
\text { Amortissement }\end{array}$ & $\begin{array}{l}\text { Moy VAN } \\
\text { SD VAN }\end{array}$ & $\begin{array}{c}\mathrm{CV}_{\mathrm{VAN}} \\
(\%)\end{array}$ \\
\hline Braffedon & $\begin{array}{c}2 \\
460155^{\mathrm{a}} \\
\pm 782992\end{array}$ & 70837 & 329456 & $\begin{array}{l}2059861^{\mathrm{a}} \\
\pm 715815\end{array}$ & 35 \\
\hline Kokou & $\begin{array}{c}2 \\
643547^{\mathrm{a}} \\
\pm 283972\end{array}$ & 87797 & 434232 & $\begin{array}{l}2121519^{\mathrm{a}} \\
\pm 290592\end{array}$ & 14 \\
\hline Lahou & $\begin{array}{c}4 \\
213997^{\mathrm{a}} \\
\pm 664807\end{array}$ & 77313 & 394272 & $\begin{array}{l}3742412^{\mathrm{a}} \\
\pm 647535\end{array}$ & 17 \\
\hline Mackey & $\begin{array}{c}1 \\
200126^{b} \\
\pm 857584\end{array}$ & 27266 & 121824 & $\begin{array}{l}1051035^{\mathrm{b}} \\
\pm 888020\end{array}$ & 84 \\
\hline Nguessandon & $\begin{array}{c}3005 \\
176^{\mathrm{a}} \\
+238468\end{array}$ & 90568 & 465962 & $\begin{array}{l}2448645^{\mathrm{a}} \\
\pm 228146\end{array}$ & 9 \\
\hline Noumouzou & $\begin{array}{c}3383 \\
257^{\mathrm{a}} \\
+332696\end{array}$ & 97675 & 516888 & $\begin{array}{l}2695254^{\mathrm{a}} \\
\pm 315444\end{array}$ & 12 \\
\hline Tioko & $\begin{array}{c}3016 \\
496^{\mathrm{a}} \\
+239537 \\
\end{array}$ & 49946 & 208872 & $\begin{array}{l}2757677^{\mathrm{a}} \\
\pm 255812\end{array}$ & 9 \\
\hline Probabilités & $\mathrm{P}<0,05$ & & & $P<0,05$ & \\
\hline MOY.LGL & $\begin{array}{r}2846108 \\
\pm 999271 \\
\end{array}$ & 71628 & 353072 & $\begin{array}{r}2410915 \\
\pm 901207 \\
\end{array}$ & 37 \\
\hline
\end{tabular}

Moy : Moyenne SD : écart type CV : Coefficient de variation VP : valeur de la production VAN : Valeur

ajoutée nette LGL : Lagune de Grand-Lahou

Les valeurs portant la même lettre en exposant ont des variances homogènes

Le tableau 2 présente les éléments qui entrent dans la détermination de la Valeur ajoutée nette annuelle. Ces chiffres indiquent la richesse monétaire réelle créée par le métier du filet maillant par village. Tout comme la valeur de la production, les VAN ne présentent aucune différence significative mis à part le village de Mackey. La VAN la plus élevée s'observe à Lahou et les plus faibles à Mackey et à Braffedon où l'on retrouve les $C V_{\text {VAN }}$ les plus élevés $\left(\mathrm{CV}_{\mathrm{VAN}}>25 \%\right)$. On constate aussi que la valeur de la production et la valeur ajoutée nette chez les professionnels au métier de filet maillant sont très proches.

Tableau 3 : Revenus du travail par village et par pêcheur

\begin{tabular}{ccccc}
\hline Villages & $\begin{array}{c}\text { Moy RT } \\
\text { SD RT }\end{array}$ & $\begin{array}{c}\mathrm{CV}_{\mathrm{RT}} \\
(\%)\end{array}$ & $\begin{array}{c}\text { Moy RTm } \\
\text { SD RTm }\end{array}$ & $\begin{array}{c}\mathrm{CV}_{\mathrm{RTm}} \\
(\%)\end{array}$ \\
\hline Braffedon & $1980768^{\mathrm{a}}$ & 36 & $165064^{\mathrm{a}}$ & 36 \\
& \pm 715151 & & \pm 59596 & \\
Kokou & $2041455^{\mathrm{a}}$ & 14 & $170121^{\mathrm{a}}$ & 14 \\
& \pm 290837 & & \pm 24236 & \\
Lahou & $3662979^{\mathrm{a}}$ & 18 & $305248^{\mathrm{a}}$ & 18 \\
& \pm 647539 & & \pm 53962 & \\
Mackey & $974028^{\mathrm{b}}$ & 81 & $81169^{\mathrm{b}}$ & 81 \\
& \pm 793622 & & \pm 66135 & \\
\hline
\end{tabular}




\begin{tabular}{ccccc}
\hline Nguessandon & $2374508^{\mathrm{a}}$ & 9 & $197876^{\mathrm{a}}$ & 7 \\
& \pm 217147 & & \pm 14179 & 11 \\
Noumouzou & $2690304^{\mathrm{a}}$ & 11 & $224192^{\mathrm{a}}$ & 10 \\
& \pm 292329 & & \pm 24361 & \\
Tioko & $2679557^{\mathrm{a}}$ & 10 & $223296^{\mathrm{a}}$ & \pm 21322 \\
& \pm 255868 & & $\mathrm{P}<0,05$ & 38 \\
\hline Probabilités & $\mathrm{P}<0,05$ & & 195281 & \pm 7527238 \\
\hline
\end{tabular}

Moy : Moyenne SD : écart type CV : Coefficient de variation VAN : Valeur ajoutée nette RT : revenu annuel du travail RTm : Revenu moyen du travail par mois

LGL : Lagune de Grand-Lahou ; Les valeurs portant la même lettre en exposant ont des variances homogènes

Le maximum du revenu mensuel estimé est relevé à Lahou. Les valeurs les plus faibles sont enregistrées à Braffedon et à Mackey. On note que le rapport des revenus moyens mensuels estimés pris par pairs n’atteint pas 2 dans la presque totalité des villages échantillonnés. Le cas de Mackey demeure l'exception avec un rapport de 3,76 entre le revenu moyen dans ce village et le revenu moyen le plus élevé à l'échelle de la lagune.

Tableau 4 : Taux de valeur ajoutée (TVA), taux de rentabilité du capital investi (TRCI), effort de pêche critique de rentabilité (eff. crit.) et coût de production du Kg de poisson

(CPKPD)

\begin{tabular}{|c|c|c|c|c|c|c|c|c|}
\hline Villages & $\begin{array}{c}\text { Moy TVA } \\
\text { SD TVA }\end{array}$ & $\begin{array}{c}\mathrm{CV}_{\text {TVA }} \\
(\%)\end{array}$ & $\begin{array}{l}\text { Moy TRCI } \\
\text { SD TRCI }\end{array}$ & $\begin{array}{c}\mathrm{CV}_{\mathrm{TRCI}} \\
(\%)\end{array}$ & $\begin{array}{l}\text { Moy eff. crit } \\
\text { SD eff. Crit. } \\
\text { (Sorties) }\end{array}$ & $\begin{array}{c}C V_{\text {eff. crit. }} \\
\text { (\%) }\end{array}$ & $\begin{array}{c}\text { Moy } \\
\text { CPKPD } \\
\text { SD } \\
\text { CPKPD } \\
\text { (FCFA/Kg) }\end{array}$ & $\mathrm{CV}$ \\
\hline Braffedon & $\begin{array}{c}0,73 \\
\pm 0,07\end{array}$ & 9 & $\begin{array}{l}1,56^{\mathrm{ab}} \\
\pm 0,72\end{array}$ & 46 & $\begin{array}{l}185^{\mathrm{a}} \\
\pm 68\end{array}$ & 37 & $\begin{array}{l}371^{\mathrm{a}} \\
\pm 43\end{array}$ & 12 \\
\hline Kokou & $\begin{array}{c}0,79 \\
\pm 0,05\end{array}$ & 7 & $\begin{array}{c}1,34^{\mathrm{b}} \\
\pm 0,42\end{array}$ & 31 & $\begin{array}{l}206^{\mathrm{a}} \\
\pm 73\end{array}$ & 36 & $\begin{array}{l}261^{\mathrm{a}} \\
\pm 43\end{array}$ & 16 \\
\hline Lahou & $\begin{array}{c}0,89 \\
\pm 0,02\end{array}$ & 2 & $\begin{array}{l}3,58^{\mathrm{cd}} \\
\pm 0,89\end{array}$ & 25 & $\begin{array}{c}74^{\mathrm{b}} \\
\pm 17\end{array}$ & 23 & $\begin{array}{l}278^{\mathrm{a}} \\
\pm 23\end{array}$ & 8 \\
\hline Mackey & $\begin{array}{c}0,85 \\
\pm 0,08\end{array}$ & 9 & $\begin{array}{l}0,85^{\mathrm{ab}} \\
\pm 1,28\end{array}$ & 151 & $\begin{array}{c}* 137^{\mathrm{ab}} \\
\pm 71\end{array}$ & $* 52$ & $\begin{array}{c}678^{\mathrm{b}} \\
\pm 632\end{array}$ & 93 \\
\hline N'guessandon & $\begin{array}{c}0,81 \\
\pm 0,04\end{array}$ & 5 & $\begin{array}{l}1,90^{\text {abc }} \\
\pm 0,80\end{array}$ & 42 & $\begin{array}{l}134^{\mathrm{ab}} \\
\pm 39\end{array}$ & 29 & $\begin{array}{l}167^{\mathrm{a}} \\
\pm 18\end{array}$ & 11 \\
\hline Noumouzou & $\begin{array}{c}0,80 \\
\pm 0,04\end{array}$ & 6 & $\begin{array}{l}2,03^{\mathrm{abc}} \\
\pm 0,22\end{array}$ & 11 & $\begin{array}{l}125^{\mathrm{ab}} \\
\pm 15\end{array}$ & 12 & $\begin{array}{l}210^{\mathrm{a}} \\
\pm 18\end{array}$ & 9 \\
\hline Tioko & $\begin{array}{c}0,91 \\
\pm 0,03 \\
\end{array}$ & 3 & $\begin{array}{r}4,07^{\mathrm{d}} \\
\pm 1,34 \\
\end{array}$ & 33 & $\begin{array}{r}69^{\mathrm{b}} \\
\pm 26 \\
\end{array}$ & 38 & $\begin{array}{r}274^{\mathrm{a}} \\
\pm 103 \\
\end{array}$ & 38 \\
\hline Probabilités & \multicolumn{2}{|c|}{$\mathrm{P}>0,05$} & \multicolumn{2}{|l|}{$\mathrm{P}<0,05$} & \multicolumn{2}{|c|}{$\mathrm{P}<0,05$} & $\mathrm{P}<0,05$ & \\
\hline MOY. LGL & $\begin{array}{l}0,84 \\
\pm 0,06 \\
\end{array}$ & 8 & $\pm 1,6$ & 76 & $\begin{array}{r}128 \\
\pm 69 \\
\end{array}$ & 54 & $\begin{array}{r}320 \\
\pm 272 \\
\end{array}$ & 85 \\
\hline
\end{tabular}

Moy : Moyenne ; SD : écart type ; CV : Coefficient de variation ; TVA: Taux de valeur ajoutée ; TRCI : Taux de rentabilité du capital investi ; eff. crit. : Effort de pêche critique de rentabilité ; LGL : Lagune de Grand-Lahou

Les valeurs portant la même lettre en exposant ont des variances homogènes ; * valeur calculée en ne tenant compteque des unités de pêche ayant une rentabilité effective 
Le taux de valeur ajoutée (TVA) varie entre 0,73 et 0,91 (tableau 4). La valeur minimale est enregistrée à Braffedon et le Taux le plus élevé à Tioko. Le test de comparaison multiple indique que le TVA est homogène aussi bien dans l'ensemble des villages échantillonnés qu'au niveau des unités de pêche observées ( $\mathrm{P}>0,05$ et $\mathrm{CV}_{\text {TVA }}<25 \%$ ). Les coefficients de variation des taux sont faibles avec un maximum de $9 \%$, indiquant que la presque totalité de la valeur de la production constitue la richesse générée par l'activité de pêche.

Selon les résultats du tableau 4, le TRCI est très variable selon les villages. Il se situe entre 0,85 (Mackey) et 4,07 (Tioko).

Le Délai de Récupération du Capital Investi (DRCI) ou encore Effort de Pêche Critique de Rentabilité (EPCR) est très variable à l'échelle de la lagune. Il équivaut approximativement à la moitié de l'année de pêche. L'EPCR enregistre ses valeurs minimales à Tioko (69 \pm 26 sorties) et à Lahou (74 \pm 17 sorties). Les unités de pêches de ces localités sont par conséquent les plus performantes de l'ensemble étudié. Ces résultats traduisent que les points morts de l'activité des unités de pêche sont atteints respectivement après 3,28 mois et 3,52 mois de pêche.

Le coût de production d'un kilogramme de poisson débarqué est maximal à Mackey et minimal à N'guessandon. Toutefois, l'analyse de comparaison multiple indique qu'il n'existe aucune différence significative entre les CPKPD calculés à l'exception du village de Mackey.

Tableau 5 : Matrice de corrélation bilatérale issue du test de rang de Spearman sur la puissance de pêche, la valeur de la production, le TRCI, le CPKPD, le nombre de personnes à charge, le niveau d'étude, l'âge, l'expérience, la valeur des investissements et le résultat

\begin{tabular}{|c|c|c|c|c|c|c|c|c|c|c|}
\hline & & $\begin{array}{c}\text { Valeur } \\
\text { production }\end{array}$ & TRCI & CPKPD & $\begin{array}{c}\text { Personnes } \\
\text { à } \\
\text { charge }\end{array}$ & $\begin{array}{l}\text { Niveau } \\
\text { d'étude }\end{array}$ & Age & Expérience & Invest. & $\begin{array}{l}\text { Résultat } \\
\text { net }\end{array}$ \\
\hline $\begin{array}{c}\text { Valeur } \\
\text { production }\end{array}$ & Corr. & 1,000 & ,626 &,$- 472^{* * * *}$ & 109, &,- 265 & 189, & 263 &, $468^{* * *}$ &, $970^{* * * *}$ \\
\hline TRCI & Corr. & & 1,000 &,- 244 &,- 143 &,- 238 &,- 230 &,- 095 &,$- 333^{*}$ & ,662 \\
\hline CPKPD & Corr. & & & 1,000 & ,000 & 108 & ,074 &,$- 329 *$ &,$- 546^{* * *}$ &,$- 543^{* * *}$ \\
\hline $\begin{array}{l}\text { Personnes à } \\
\text { charge }\end{array}$ & Corr. & & & & 1,000 &,- 208 & ,330* & $384^{* *}$ & ,075 & ,047 \\
\hline $\begin{array}{l}\text { Niveau } \\
\text { d'étude }\end{array}$ & Corr. & & & & & 1,000 &,$- 366^{* *}$ &,- 265 & 146 &,- 211 \\
\hline Age & Corr. & & & & & & 1,000 &, $518^{* * * *}$ & ,224 & ,138 \\
\hline $\begin{array}{l}\text { Expérience } \\
\text { Invest. } \\
\text { Résultat net }\end{array}$ & $\begin{array}{l}\text { Corr. } \\
\text { Corr. } \\
\text { Corr. }\end{array}$ & & & & & & & 1,000 & $\begin{array}{l}, 350^{* * *} \\
1,000\end{array}$ & $\begin{array}{l}, 243 \\
, 497^{* * *} \\
1,000\end{array}$ \\
\hline
\end{tabular}

Corr. : Coefficient de corrélation

*. La corrélation est significative au niveau 0,1 (bilatéral).

**. La corrélation est significative au niveau 0,05 (bilatéral).

***. La corrélation est significative au niveau 0,01 (bilatéral). 
Le tableau 5 montre qu'il existe plusieurs corrélations significatives entre les variables considérées. Il ressort que la valeur de la production est positivement corrélée à l'investissement mobilisé pour l'acquisition des engins de capture. Ce paramètre, équivalent du chiffre d'affaire est fortement corrélé au TRCI et à la valeur des investissements. Cette relation signifie que plus la valeur de la production est grande et plus on accroit le taux de récupération du capital investi. La valeur de la production et le résultat net sont aussi une fonction croissante de l'investissement. A l'inverse, le coût de production du kilogramme de poisson débarqué est négativement corrélé à ces 3 facteurs. Cela signifie que le coût de production diminue lorsqu'ils augmentent.

Par ailleurs, le TRCI et le CPKPD sont négativement corrélés à la valeur des investissements. Autrement, ce lien révèle que l'investissement pourraient avoir des effets contreproductifs sur les performances économiques des unités de pêche même si elles réduisent par la même occasion le coût de production du kilogramme de poisson débarqué.

La réduction du CPKPD sous l'influence des investissements croissants est soutenue par la corrélation très significative entre l'investissement et la valeur de la production. L'investissement impacte positivement les quantités débarquées et la valeur de la production. En outre, plus on investit et plus on réduit le taux de récupération du capital investi, c'est dire que moins le rapport entre la résultat net et l'investissement est élevé. Il existerait alors un niveau d'investissement qui nuirait à la performance économique des unités de pêche.

Les variables sociales que sont l'âge du pêcheur, son niveau d'étude et le nombre de personnes à sa charge ne présentent aucune corrélation significative avec les variables de rentabilité et de performance économique. On observe que les pêcheurs les plus expérimentés ont un investissement plus élevé. En conséquence, elles accroissent les productions et réduisent ainsi le CPKPD.

Pour des nécessités de comparaison, le tableau 6 consigne les paramètres de production de performances de 5 activités agricoles et 2 activités aquacoles qui sont les alternatives économiques à la pêche artisanale dans la zone de Grand-Lahou.

Parmi toutes les spéculations considérées, nous en dénombrons 4 dont les valeurs de production annuelles sont supérieures à la VP moyenne estimée au niveau de la lagune. Ce sont par ordre décroissant : les cultures de tomate, d'oignon, la production de pépinière d'hévéa et de la culture piment. Ce sont les silures qui sont susceptibles de produire un chiffre d'affaire de 3 125000 FCFA par cycle de 10 mois. Parmi toutes ces activités alternatives, seules les 3 premières cultures citées ont un résultat net supérieur à celui des unités de pêche. Toutefois, elles devraient être menées en production 
intensive avec un investissement variant entre 2,2 et 3 fois l'investissement moyen de l'activité de pêche artisanale pour un rapport des résultats nets fluctuant entre 1,47 et 2 . Cela requiert par ailleurs un niveau de technicité suffisamment élevé pour conduire correctement les itinéraires techniques et l’existence d'un marché favorable qui rémunère entièrement les facteurs de production.

Tableau 6 : Paramètres de productions et de performances économiques de référence dans la zone de Grand- Lahou

\begin{tabular}{|c|c|c|c|c|c|c|c|c|c|c|c|c|}
\hline $\begin{array}{l}\text { Activit } \\
\text { é de } \\
\text { produc } \\
\text { tion }\end{array}$ & $\begin{array}{c}\text { Mode } \\
\text { de } \\
\text { produc } \\
\text { tion }\end{array}$ & $\begin{array}{c}\text { Dimen } \\
\text { sion }\end{array}$ & $\begin{array}{c}\text { Cycle } \\
\text { s/an }\end{array}$ & $\begin{array}{c}\text { Prix } \\
\text { moy } \\
\text { en }\end{array}$ & $\begin{array}{c}\text { Rende } \\
\text { ment }\end{array}$ & $\begin{array}{c}\text { VP } \\
\text { (FCF } \\
\text { A) }\end{array}$ & $\begin{array}{c}\mathrm{RN} \\
\text { (FCF } \\
\mathrm{A})\end{array}$ & $\begin{array}{c}\text { VAN } \\
\text { (FCF } \\
\text { A) }\end{array}$ & $\begin{array}{c}\text { TV } \\
\text { A }\end{array}$ & $\begin{array}{c}\mathrm{Rm} \\
(\mathrm{FCF} \\
\mathrm{A})\end{array}$ & $\begin{array}{l}\text { TR } \\
\text { CI }\end{array}$ & $\begin{array}{c}\text { CP } \\
\text { U }\end{array}$ \\
\hline \multirow[t]{2}{*}{ tilapia } & Intensi & 5 & 1 & 150 & 225 & 1687 & 277 & 277 & 0,2 & 70 & 0,1 & 125 \\
\hline & f & $\begin{array}{l}\text { étangs } \\
\text { de } \\
400 \mathrm{~m}^{2}\end{array}$ & & $\begin{array}{c}0 \\
\mathrm{~F} / \mathrm{K} \\
\mathrm{g}\end{array}$ & $\begin{array}{c}\mathrm{Kg} / \mathrm{Eta} \\
\mathrm{ng}\end{array}$ & 500 & 500 & 500 & 1 & 437 & 9 & $\begin{array}{c}5 \\
\mathrm{~F} / \mathrm{K} \\
\mathrm{g}\end{array}$ \\
\hline \multirow[t]{2}{*}{ silure } & Intensi & 5 & 1 & 100 & 625 & 3125 & 1265 & 1265 & 0,4 & 194 & 0,6 & 595 \\
\hline & f & $\begin{array}{c}\text { étangs } \\
\text { de } 400 \\
\mathrm{~m}^{2}\end{array}$ & & $\begin{array}{c}0 \\
\mathrm{~F} / \mathrm{K} \\
\mathrm{g}\end{array}$ & $\begin{array}{c}\mathrm{Kg} / \text { Eta } \\
\text { ng }\end{array}$ & 000 & 000 & 000 & 4 & 125 & 8 & $\begin{array}{c}\mathrm{F} / \mathrm{K} \\
\mathrm{g}\end{array}$ \\
\hline \multirow{4}{*}{$\begin{array}{l}\text { Pép. } \\
\text { hévéa }\end{array}$} & Intensi & 1 ha & 1 & 300 & 20000 & 6150 & 3453 & 5373 & 0,8 & 317 & 1,2 & 45 \\
\hline & f & & & $\begin{array}{c}\mathrm{F} / \mathrm{pl} \\
\text { ant }\end{array}$ & $\begin{array}{c}\text { Plants/ } \\
\text { ha }\end{array}$ & 000 & 400 & 400 & 7 & 783 & 8 & $\begin{array}{c}\mathrm{F} / \mathrm{pl} \\
\text { ant }\end{array}$ \\
\hline & Intensi & 1 ha & 2 & 350 & 8000 & 5600 & 1046 & 2782 & 0,4 & 120 & 0,1 & 190 \\
\hline & f & & & $\mathrm{F} / \mathrm{K}$ & $\mathrm{Kg} / \mathrm{ha}$ & 000 & 426 & 426 & 8 & 535 & 9 & $\mathrm{~F} / \mathrm{K}$ \\
\hline \multirow[t]{2}{*}{ Piment } & $\begin{array}{c}\text { Extens } \\
\text { if }\end{array}$ & 1 ha & 1 & $\begin{array}{c}\mathrm{g} \\
225 \\
\mathrm{~F} / \mathrm{K} \\
\mathrm{g}\end{array}$ & $\begin{array}{c}4500 \\
\mathrm{Kg} / \mathrm{ha}\end{array}$ & $\begin{array}{c}1012 \\
500\end{array}$ & $\begin{array}{l}165 \\
088\end{array}$ & $\begin{array}{c}7577 \\
13\end{array}$ & $\begin{array}{c}0,7 \\
5\end{array}$ & $\begin{array}{c}57 \\
514\end{array}$ & $\begin{array}{c}0,1 \\
6\end{array}$ & $\begin{array}{c}\mathrm{g} \\
188 \\
\mathrm{~F} / \mathrm{kg}\end{array}$ \\
\hline & Intensi & 1 ha & 2 & 170 & 20000 & 6800 & 2557 & 4498 & 0,6 & 2172 & 0,4 & 85 \\
\hline \multirow[t]{3}{*}{$\begin{array}{c}\text { Oigno } \\
\text { n }\end{array}$} & f & & & $\begin{array}{c}\mathrm{F} / \mathrm{K} \\
\mathrm{g}\end{array}$ & $\mathrm{Kg} / \mathrm{ha}$ & 000 & 294 & 994 & 6 & 74 & 7 & $\begin{array}{c}\mathrm{F} / \mathrm{K} \\
\mathrm{g}\end{array}$ \\
\hline & $\begin{array}{c}\text { Extens } \\
\text { if }\end{array}$ & 1 ha & 1 & $\begin{array}{l}125 \\
F / K\end{array}$ & $\begin{array}{c}12000 \\
\mathrm{Kg} / \mathrm{ha}\end{array}$ & $\begin{array}{c}1500 \\
000\end{array}$ & $\begin{array}{c}5962 \\
63\end{array}$ & $\begin{array}{c}1170 \\
000\end{array}$ & $\begin{array}{c}0,7 \\
8\end{array}$ & $\begin{array}{c}1293 \\
77\end{array}$ & $\begin{array}{c}0,3 \\
9\end{array}$ & $\begin{array}{l}105 \\
F / K\end{array}$ \\
\hline & $\begin{array}{c}\text { Intensi } \\
\mathrm{f}\end{array}$ & 1 ha & 2 & $\begin{array}{c}g \\
275 \\
F / K\end{array}$ & $\begin{array}{l}14000 \\
\mathrm{Kg} / \mathrm{ha}\end{array}$ & $\begin{array}{c}7700 \\
000\end{array}$ & $\begin{array}{c}2162 \\
000\end{array}$ & $\begin{array}{c}4389 \\
000\end{array}$ & $\begin{array}{c}0,5 \\
7\end{array}$ & $\begin{array}{c}1570 \\
00\end{array}$ & $\begin{array}{c}0,5 \\
3\end{array}$ & $\begin{array}{c}\mathrm{g} \\
90 \\
\mathrm{~F} / \mathrm{K}\end{array}$ \\
\hline $\begin{array}{c}\text { Tomat } \\
\text { e }\end{array}$ & $\begin{array}{c}\text { Extens } \\
\text { if }\end{array}$ & 1 ha & 1 & $\begin{array}{c}\mathrm{g} \\
125 \\
\mathrm{~F} / \mathrm{K}\end{array}$ & $\begin{array}{c}9500 \\
\mathrm{Kg} / \mathrm{ha}\end{array}$ & $\begin{array}{c}1187 \\
500\end{array}$ & $\begin{array}{c}7018 \\
73\end{array}$ & $\begin{array}{c}8966 \\
03\end{array}$ & $\begin{array}{c}0,7 \\
5\end{array}$ & $\begin{array}{c}57 \\
704\end{array}$ & $\begin{array}{c}0,2 \\
1\end{array}$ & $\begin{array}{c}\mathrm{g} \\
104 \\
\mathrm{~F} / \mathrm{K}\end{array}$ \\
\hline Riz & $\begin{array}{c}\text { Intensi } \\
\mathrm{f}\end{array}$ & 1 ha & 2 & $\begin{array}{c}\mathrm{g} \\
225 \\
\mathrm{~F} / \mathrm{K}\end{array}$ & $\begin{array}{c}5500 \\
\mathrm{Kg} / \mathrm{ha}\end{array}$ & $\begin{array}{c}2475 \\
000\end{array}$ & $\begin{array}{c}7839 \\
72\end{array}$ & $\begin{array}{c}1633 \\
500\end{array}$ & $\begin{array}{c}0,6 \\
6\end{array}$ & $\begin{array}{c}1144 \\
50\end{array}$ & $\begin{array}{c}0,4 \\
6\end{array}$ & $\begin{array}{c}\mathrm{g} \\
85 \\
\mathrm{~F} / \mathrm{K}\end{array}$ \\
\hline $\begin{array}{l}\text { bas- } \\
\text { fond }\end{array}$ & $\begin{array}{c}\text { Extens } \\
\text { if }\end{array}$ & 1 ha & 2 & $\begin{array}{c}\mathrm{g} \\
225 \\
\mathrm{~F} / \mathrm{K}\end{array}$ & $\begin{array}{c}3000 \\
\mathrm{Kg} / \mathrm{ha}\end{array}$ & $\begin{array}{c}1350 \\
000\end{array}$ & $\begin{array}{c}3582 \\
00\end{array}$ & $\begin{array}{c}1093 \\
500\end{array}$ & $\begin{array}{c}0,8 \\
1\end{array}$ & $\begin{array}{c}8116 \\
5\end{array}$ & $\begin{array}{c}0,3 \\
6\end{array}$ & $\begin{array}{c}\mathrm{g} \\
92 \\
\mathrm{~F} / \mathrm{K}\end{array}$ \\
\hline
\end{tabular}

Source : Comptes d'exploitation des services du Ministère de l'agriculture et du Ministère de la production animale et des ressources animales (référence : an 2011) ; Calculs personnels.

VP : Valeur de la Production RN : Résultat Net VAN : Valeur Ajoutée Nette Rm : revenu mensuel estimée TVA : Taux de Valeur Ajoutée TRCI : Taux de Récupération du Capital Investi CPU : Coût de Production Unitaire FCFA : Franc de la Communauté Financière Africain 


\section{Discussion}

Une étude similaire menée à Hann au sénégal par Kébé (1997) sur des unités de pêche au filet dormant en mer montre qu'un équipage de 4 pêcheurs rapportait annuellement environ 6,1 million de FCFA de chiffre d'affaire pour un résultat net de 3,5 million de FCFA. Par ailleurs, le TRCI de 1,36 est inférieur à celui obtenu en lagune de Grand-Lahou. Le niveau de rentabilité de l'activité de pêche artisanale s'explique par le fait que la demande des produits de pêche est très forte dans la majorité des régions environnant la zone de Grand-Lahou compte tenu de l'inaccessibilité et la cherté des autres formes de protéines animales telles que les viandes de bovin, de volaille et d'ovin (Weigel, 1989 ; Romagny et al., 2001 ; MINAGRI et INS, 2011). D'une année à une autre, le niveau des prix aux débarcadères de la lagune s'ajuste assez facilement au comportement du marché urbain, notamment celui d'Abidjan offrant des prix rémunérateurs aux pêcheurs (Amon-Kothias, 1981). En outre, les faibles résultats nets enregistrés à Mackey trouveraient leur explication dans la poly activité des pêcheurs sélectionnés dans cette localité et à la probable pauvreté des aires de pêche dédiées aux pêcheurs de cette localité. En effet, contrairement aux autres villages stations, l'agriculture y représente une activité de choix qui absorbe beaucoup de temps et de moyens financiers (ANADER, 2008).

Le revenu moyen de l'activité de pêche par mois à l'échelle de la lagune vaut 3,25 fois le salaire minimum interprofessionnel garanti (SMIG), 5,42 fois le salaire minimum agricole garantie (Journal officiel, 2014) et 3,82 fois le revenu mensuel brut par habitant en Côte d'Ivoire (Banque Mondiale, 2012). Seules les activités de production intensive de pépinière d'hévéa, d'oignon et de tomate procurent un revenu mensuel prévisionnel plus élevé que l'activité de pêche artisanale. Ce niveau de revenu peut s'expliquer également par le fait que la valeur monétaire du poisson auto consommé est délibérément intégré à la valeur de la production. Il est aussi important de considérer le caractère quasi individuel de la pêche sur cette lagune qui écarte le système de partage des revenus tel que cela se fait pour la pêche collective en mer (Charles-Dominique, 1990 ; Chaboud et kébé, 1986 ; Kébé, 1997 ; Kébé et Bakhayokho, 1992).

Hormis le TVA obtenu en production de pépinière d'hévéa $(0,87)$, on observe un TVA très élevé $(0,84)$ pour l'activité de pêche au filet maillant. Ce constat traduit la dominance de la productivité du travail par rapport à celle du capital immobilisé ou circulant dans la constitution de la richesse créée en pêche artisanale. Les consommations intermédiaires et l'amortissement des immobilisations sont alors faibles (Michaelof et Bridier, 1995). Cette observation est confirmée par les TVA calculés pour les cultures en mode extensif. 
En moyenne, les pêcheurs aux filet maillant récupèrent un résultat net au moins équivalent à 2 fois le capital qu'ils investissent dans l'acquisition des engins et de la pirogue. Toutefois, les gros risques qu'ils encourent jugulent cette capacité à faire du profit. En effet, cette rentabilité peut être considérablement réduite en cas de vol ou de perte d'un filet maillant. Ce taux se justifierait par le niveau élevé des investissements effectués par les unités de pêche. Paradoxalement, le TRCI serait indépendant de l'expérience du pêcheur. Ce constat signifierait qu'au-delà de 10 ans d'expérience, la technicité des pêcheurs n'influencerait plus significativement leur performance économique. En effet, il pourrait s'agir d'un effet de saturation de la technicité à long terme. Alors, il ne leur resterait autre choix que d'intensifier les investissements dans le matériel de capture. L'analyse des TRCI calculés pour les autres spéculations végétales et animales révèle des valeurs nettement inférieures à celle de la pêche en lagune de Grand-Lahou. Parmi elles, seule la production de pépinière d’hévéa donne un TRCI sensiblement supérieur à 1 .

En outre, plus on investit et plus on réduit le taux de récupération du capital investi. Il existerait alors un niveau d'investissement qui nuit à la performance économique des unités de pêche. Une des raisons pourrait être la zonation des aires de pêche par village (Anoh, 2006). En effet, il est possible que certaines zones de pêche soient plus riche en poisson que d'autres, ce qui demanderait moins d'engins pour atteindre les performances des zones moins riches. C'est le cas de la localité de Tioko dont la proximité avec la rivière Boubo semble créer un hydro système favorable à une forte densité d'espèces aussi bien saumâtres que continentales.

Quant au CPKPD, il est moins élevé que ceux des productions aquacoles de référence. Ce coût unitaire se justifie par le niveau tout aussi bas des coûts de production comparativement aux importants investissements nécessaires en aquaculture (Anonyme, 2009).

\section{Conclusion}

L'activité des unités de pêche professionnelles au filet maillant en lagune de Grand-Lahou est rentable dans tous les secteurs de la lagune. Elle se caractérise par des performances économiques relativement élevées comparativement aux alternatives agricoles et aquacoles de la zone d'étude. Au regard des sept activités agropastorales ayant servi de références, ce sont la culture de piment et la production de pépinière d’hévéa qui affichent un résultat net supérieur à l'activité de pêche artisanale des professionnels au filet maillant. Par conséquent, cette activité est compétitive. Le revenu moyen du travail estimé vaut 3,25 fois le salaire minimum interprofessionnel garanti et 5,42 fois le salaire minimum agricole garanti. Par ailleurs, le taux de valeur ajoutée, proche de 1 , indique que la productivité du facteur travail 
domine nettement celle du facteur capital sous toutes ses formes. Le pêcheur récupère en moyenne 2 fois le capital qu'il investit dans l'activité par an pendant que la quasi-totalité des activités concurrentes considérées n’atteint pas le seuil de 1. Il ressort également que l'expérience n’influence pas significativement les performances financières chez les pêcheurs de plus de 10 ans d'ancienneté. La technicité serait ainsi plafonnée avant que le pêcheur n’ait atteint 10 ans d'expérience. La recherche de la performance se fait alors par l'investissement dont les effets deviennent contreproductifs à cause du suréquipement. Enfin, Tioko s’est révélé comme le village le plus performant du système d'enquête. Le coût de production du kilogramme de poisson débarqué est relativement plus élevé que le Prix de revient unitaire des spéculations agropastorales de références.

\section{References:}

1. Aktouf, O. (1987). Méthodologie des sciences sociales et approche qualitative des organisations : une introduction à la démarche classique et une critique. Presses de l'Université du Québec, HEC Presses.

2. Allison, E. \& Ellis, F. (2001). The livelihoods approach and management of small-scale fisheries. Marine Policy, 25 :377-388.

3. Amon-Kothias, J. B. (1981). La consommation de poisson frais en lagune Ebrié (Côte d'ivoire). Doc. Sci. Centr. rech. Océanogr. Abidjan, Vol. XII, N² : 1-27.

4. ANADER (2008). Rapport d'activités annuel sur l'agriculture, l'élevage et la pêche dans la zone de Grand-Lahou. Document ANADER.

5. Anoh, K. P. (2007). Impact environnemental et socio-économique de la pêche par empoisonnement en milieu littoral ivoirien. Revue de géographie tropicale et d'environnement 2 : 3-13.

6. Anoh, K. P. (2010). Stratégies comparées de l'exploitation des plans d'eau lagunaire de Côte-d'Ivoire. Les Cahiers d'Outre-Mer, 251, 347-363.

7. Anonyme (2009). Appui à la gestion de pêche artisanale transfrontalière: Etude diagnostic de la pêcherie de mulets sur la grande côte du Sénégal. Direction des pêches Maritimes, Dakar.

8. Anonyme (2013). Décret $n^{\circ} 2013-791$ du 20 novembre 2013 portant revalorisation $d u$ salaire minimum interprofessionnel garanti. République de Côte d'Ivoire.
9. Banque
Mondiale
(2012).
Disponible
à
http://donnees.banquemondiale.org/indicateur/NY.GNP.PCAP.CD (consulté le 12 juin 2016)

10. Bleu (2015). Contamination des poissons par les métaux lourds : Cas 
de cinq espèces de la lagune de Grand-Lahou. Thèse de doctorat de l'Université de Nangui Abrogoua, Côte d'Ivoire

11. Bridier, M., \& Michailof, S. (1995). Guide pratique d'analyse des projets, évaluation et choix des projets d'investissement, $5^{\mathrm{e}}$ édition, Economica, Paris.

12. Chaboud, C., \& Kébé, M. 1986. Les aspects socioéconomiques de la pêche artisanale maritime au Sénégal : Mutations technologiques et politiques de développement. ORSTOM, CRODT/ISRA, Dakar.

13. Charles-Dominique, E. (1993). L'exploitation de la lagune Aby (Côte d'lvoire) par la pêche artisanale : dynamique des ressources, de l'exploitation et des pêcheries. Thèse de doctorat, Université de Montpellier, France.

14. Charles-Dominique, E., Ecoutin, J. M., \& San Gnanmilin, A. (1980). La pêche artisanale en lagune Aby-Tendo-Ehy (Côte d'Ivoire) : premières estimations de la production. Arch. Scient. Centr. Rech. Océanogr., Abidjan, 6 (4) : 1-12.

15. FAO (2014). La situation mondiale des pêches et de l'aquaculture. Disponible à http://www.fao.org/news/story/fr/item/231537/icode/ (consulté le 12/05/2016)

16. Francini-Filho, R. B., \& De Moura, R. L. (2008). Dynamics of fish assemblages on coral reefs subjected to different management regimes in the Abrolhos Bank, eastern Brazil. Aquatic Conserv: Mar. Freshw. Ecosyst. 18 : 1166-1179

17. Gérard, M., \& Gréber, P. (1985). Analyse de la pêche artisanale au Cap vert : description et étude critique du système d'enquête. Doc. Scient. Cent. Rech. Océanogr. Dakar-Thiaroye.

18. Kébé, M. (1997). Etude des coûts et revenus des unités de pêche artisanale à Han, Sénégal. Programmes pour le développement intégré des pêches artisanales en Afrique de l'Ouest, Cotonou, Bénin, DIPA/WP/103, 20 p.

19. Kébé, M., \& Horeman, B. (1998). Guide méthodologique pour l'étude des coûts et revenus en pêche artisanale en Afrique. Programmes pour le développement intégré des pêches artisanales en Afrique de l’Ouest, Cotonou, Bénin.

20. Kébé, M., \& Bakhayokho, M. (1992). Étude technico-économique comparative de la pêche à la palangre améliorée utilisant le vireligne et de la pêche à la palangre traditionnelle. ISRA, Cahiers d’information, vol $6 \mathrm{~N}^{\circ} 1$, Dakar.

21. Kouakou, R., Kouassi, A. M., Kouassi, E., Kwa-Koffi, Etile, N. R., \& Trokourey, A. (2015). Distribution of organochlorine pesticides and polychlorinated biphenyls in the sediments of a tropical lagoon 
(The Grand-Lahou lagoon, Côte d'Ivoire). Journal of Applied Biosciences, 88 :8167- 8179.

22. Laë, R. (1992). Les pêcheries artisanales lagunaires ouest africaines : échantillonnage et dynamique de la ressource et de l'exploitation. Thèse de Doctorat de l'Université de Bretagne Occidentale, Paris, France.

23. Levèque, C., Paugy, D., \& Teugels, G. G. (1992). Faunes des poissons d'eaux douces et saumâtres d'Afrique de l'Ouest. In : Faune tropicale, vol. 28, éditions ORSTOM, Paris.

24. Levrel, H., Hay, J., Bas, A., Gastineau, P., \& Pioch, S. (2012). Coût d'opportunité versus coût du maintien des potentialités écologiques : deux indicateurs économiques pour mesurer les coûts de l'érosion de la biodiversité. Natures, sciences et société, 20 : 1629.

25. McClanahan, T. R., Maina, J., \& Davies, J. (2005). Perceptions of resource users and managers towards fisheries management options in Kenyan coral reefs. Fisheries. Management and Ecology, 12:105112.

26. MINAGRI \& INS (2011). Rapport sur la dynamique de la consommation alimentaire en Côte d'Ivoire. RESAKSS, Michigan State University et Syngeta, Ministère de l'agriculture, Institut National de la Statistique, Côte d'Ivoire.

27. Paugy, D., Lévêque, C., \& Teugels, G. G. (2004). Poissons d'eaux douces et saumâtres de l'Afrique de l'Ouest. IRD éditions/MRAC, tome 3.

28. Pauly, D. (1990). On Malthusian overfishing. Naga, the ICLARM Quarterly. 13: 3-4.

29. Pauly, D., Christensen, V., Guenette, S., Pitcher, T. J., Sumaila, U. R., Walters, C. J., Watson, R., \& Zeller, D. (2002). Towards sustainability in world fisheries. Nature, 418 :689-695.

30. Polunin, N. V. C., Roberts, C. M., \& Pauly, D. (1996). Developments in tropical reef fisheries science and management. In Reef Fisheries, Vol. 20, Polunin, N.V.C., C.M. Roberts (eds). Chapman \& Hall, London.

31. Romagny, B., Menard, F., Dewals, P., Gaertner, D., \& N'Goran, N. (2001). Le "faux-poisson" d'Abidjan et la pêche sous DCP dérivants dans l'Atlantique tropical Est : circuit de commercialisation et rôle socio-économique. DCP, sociétés et systèmes halieutiques, Montpellier, 634-652.

32. Sankaré, Y., Kaba, N., \& Ettien, N. (1994). La pêche par empoisonnement dans les eaux saumâtres tropicales (lagunes 
ivoiriennes) : effets sur l'environnement. Document scientifique CRO Abidjan, Côte d'Ivoire.

33. Turay, F., \& Verstralen, K. (1997). Coûts et revenus en pêche artisanales: méthodologie et leçons retenues des études de cas. Programmes pour le développement intégré des pêches artisanales en Afrique de l'Ouest, Cotonou, Bénin.

34. UEMOA (2013). La pêche continentale dans les Etats membres de l'UEMOA : Enquête-cadre 2012. IRD-AgroCampus-Oceanic, Disponible à http://atlas.statpeche-uemoa.org, consulté le 14/04/16.

35. Weigel, J. Y. (1989). La commercialisation du poisson en pays lagunaire ivoirien. Thèses de l'Institut Français de la Recherche Scientifique, du développement et de la Coopération, France.

36. Zar, J. H. (1999). Bio statistical Analysis. 4th Edition. Prentice-Hall, Englewood Cliffs, New Jersey. 